\title{
Shielding of Transient Electromagnetic Signals by a Thin Conducting Sheet ${ }^{1}$
}

\author{
N. R. Zitron 2
}

(March 2, 1960)

\begin{abstract}
The shielding effect of a thin, horizontal imperfectly conducting sheet against the transient field of a vertical magnetic dipole when excited by a ramp function is investigated. The results are calculated by taking Laplace transforms of the frequency spectrum functions for the steady-state problem. The response to the ramp function is calculated and the significance of the results in shielding against surges is discussed.
\end{abstract}

\section{Introduction}

The problem of shielding electrical equipment from transient signals emitted by highpowered radio transmitters is of practical interest. The response of a physical system to a transient electromagnetic excitation is usually in the form of a surge which decays after some time, leaving the steady fields. This paper deals with the shielding effect of a thin, infinite sheet of imperfectly conducting material against such surges.

Wait $[1]^{3}$ has considered this problem in terms of a magnetic dipole excited by a step function. The purpose of this paper is to simulate more closely the building up of a surge by considering an excitation in the form of a ramp function. The ramp function takes into account the rise time of the signal, that is, the time required for the signal to rise from its initial value of zero to its peak value.

Wait [1] has calculated the response to a step function by taking Laplace transforms of the frequency spectrum functions obtained previously by a low-frequency approximation. He then suggested that the response to an arbitrary excitation may be obtained by means of the superposition theorem. Although the superposition theorem is elegant, it does not appear to offer any practical advantage over the transform method in the present problem, so the transform method is employed here.

\section{Statement of the Problem}

The situation considered is the excitation by a ramp function of a vertically oriented magnetic dipole above a thin, imperfectly conducting sheet. The object is to calculate the transient fields on the opposite side of the sheet with the aid of the frequency spectrum functions obtained previously by Wait [2, 3].

The magnetic dipole source is a loop antenna situated at the origin of a cylindrical coordinate system $(\rho, \phi, z)$. The axis of the loop of area $d A$ (and of the dipole) is oriented in the $z$ direction and has a total current $I$. The conducting sheet is centered in the plane $z=-a$ (fig. 1). Its thickness is $d$ and its conductivity is $\sigma$.

The spectrum functions employed here are based upon a low-frequency approximation and, therefore, the distances $a, \rho$, and $z$, are assumed to be small compared to the wavelength. It is assumed also that $d<<a$.

The low-frequency approximation, which restricts these considerations to thin, conducting sheets, was employed by Wait [2] to obtain the electric and magnetic fields as functions of frequency in order to simplify some extremely complicated expressions [3]. Once these

1 Contribution from Gordon McKay Laboratory, Harvard University, Cambridge, Mass. This work was supported by AFWSP under Contract Nonr 1866(26) between the Office of Naval Research and Harvard University.

3 Present address: Division of Engineering, Brown University, Providence $12, \mathrm{R}$. 


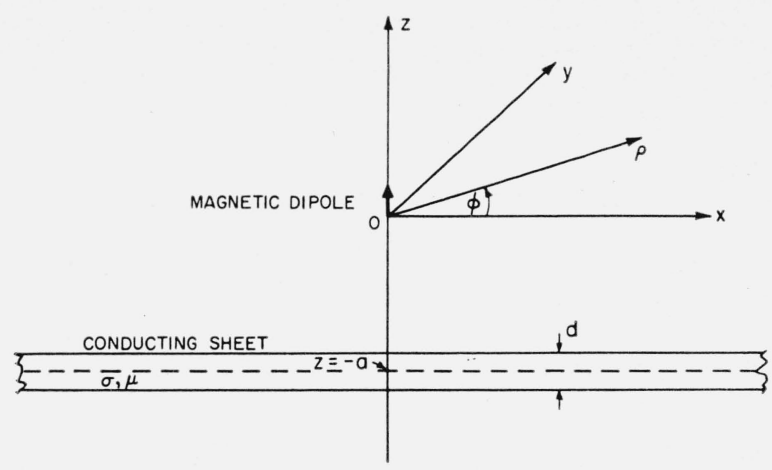

Figure 1. Shielded region.

fields have been obtained in the frequency domain, they are in accord with the material conditions and they may then be transformed into the time domain.

The spectrum functions for a harmonic dipole source having time dependence $e^{i \omega t}$ have been obtained by Wait [1]. The spectrum of the magnetic field is expressed as

$$
H(i \omega)=-\operatorname{grad} \psi(i \omega)=\left[H_{\rho}(i \omega), 0, H_{z}(i \omega)\right]
$$

where the magnetic potential $\psi(i \omega)$ is given by

$$
\psi(i \omega)=b\left[\frac{I(i \omega) z}{\left(z^{2}+\rho^{2}\right)^{3 / 2}}+I(i \omega) i \omega \frac{\partial}{\partial z} \int_{0}^{\infty} \frac{e^{\lambda z} J_{0}(\lambda \rho)}{\lambda / \alpha+i \omega} d \lambda\right]
$$

where $b=d A / 4 \pi$ and $\alpha=\sigma \mu d / 2$ where $\mu=4 \pi \times 10^{-7} \mathrm{~h} / \mathrm{m}$.

It is appropriate at this point to differentiate under the integral sign in eq (2) in order to avoid the subsequent occurrence of an integral that does not exist. Equation (2) then becomes

$$
\psi(i \omega)=b\left[\frac{I(i \omega) z}{\left(z^{2}+\rho^{2}\right)^{3 / 2}}+I(i \omega) i \omega \int_{0}^{\infty} \frac{\lambda e^{\lambda z} J_{0}(\lambda \rho)}{\lambda / \alpha+i \omega} d \lambda\right]
$$

The frequency spectrum of the electric field is

$$
E_{\varphi}(i \omega)=-b u I(i \omega) i \omega\left[\frac{\rho}{\left(z^{2}+\rho^{2}\right)^{3 / 2}}+i \omega \frac{\partial}{\partial \rho} \int_{0}^{\infty} \frac{e^{\lambda z} J_{0}(\lambda \rho)}{\lambda / \alpha+i \omega} d \lambda\right] .
$$

The frequency spectrum of the current $I(i \omega)$ is the Laplace transform of the excitation $j(t)$. It is desirable for many applications to study the excitation occurring in a radio transmitter. When a transmitter is switched on, the signal is initially zero. It rises to its peak value after a certain time which will be called the rise time of the signal. It is convenient to simulate this excitation by means of a ramp function (fig. 2) which is defined as

$$
j(t)=\frac{I_{0}}{\tau}[t u(t)-(t-\tau) u(t-\tau)]
$$

where $u(t)$ is the unit-step function

$$
u(t)= \begin{cases}0 & t<0 \\ 1 & t>0\end{cases}
$$

$I_{0}$ is the peak value of the current and $\tau$ is the rise time.

Figure 2. The ramp function.

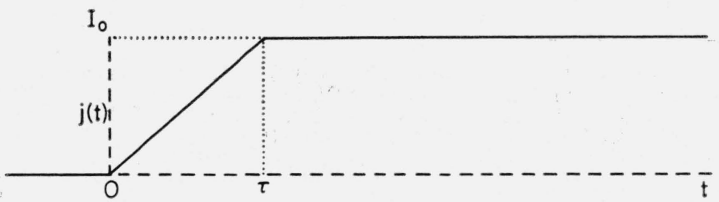


The current spectrum of the ramp function is

$$
I(i \omega)=\int_{0}^{\infty} j(t) e^{-i w t} d t=\frac{I_{0}}{\tau}\left[\frac{1}{(i \omega)^{2}}\left(1-e^{-i \omega \tau}\right)\right] .
$$

The spectrum functions of the magnetic potential (eq (3)) and the electric field (eq (4)) can now be written explicitly in terms of the frequency as follows:

and

$$
\psi(i \omega)=b \frac{I_{0}}{\tau}\left(1-e^{-i \omega \tau}\right)\left[\frac{1}{(i \omega)^{2}} \frac{z}{\left(z^{2}+\rho^{2}\right)^{3 / 2}}+\frac{1}{i \omega} \int_{0}^{\infty} \frac{\lambda^{e^{\lambda z}} J_{0}(\lambda \rho)}{\lambda / \alpha+i \omega} d \lambda\right]
$$

$$
E_{\phi}(i \omega)=b u \frac{I_{0}}{\tau}\left(1-e^{-i \omega \tau}\right)\left[\frac{1}{i \omega} \frac{z}{\left(z^{2}+\rho^{2}\right)^{3 / 2}}+\frac{\partial}{\partial \rho} \int_{0}^{\infty} \frac{e^{\lambda z} J_{0}^{(\lambda \rho)}}{\lambda / \alpha+i \omega} d \lambda\right]
$$

The transient response to the ramp function is calculated by taking the inverse Laplace transform of the frequency spectrum. The transform over the frequency domain yields the behavior of the fields in the time domain. The inverse transforms $\psi(t)$ and $e_{\phi}(t)$ are defined by

and

$$
\psi(t)=\frac{1}{2 \pi i} \int_{c-i \infty}^{c+i \infty} \psi(i \omega) e^{i \omega t} d(i \omega)
$$

$$
e_{\phi}(t)=\frac{1}{2 \pi i} \int_{c-i \infty}^{c+i \infty} E_{\phi}(i \omega) e^{i \omega t} d(i \omega)
$$

The spectrum functions (eqs (7) and (8)) are transformed respectively into

$\psi(t)-b I_{0}\left[\frac{z}{\left(z^{2}+\rho^{2}\right)^{3 / 2}} \frac{1}{\tau}(t u(t)-(t-\tau) u(t-\tau))+\frac{1}{\tau / \alpha}\left\{\frac{u(t)-u(t-\tau)}{\left(z^{2}+\rho^{2}\right)^{3 / 2}}-\frac{u(t)}{\left((-z+t / \alpha)^{2}+\rho^{2}\right)^{3 / 2}}\right.\right.$

and

$$
\left.\left.+\frac{u(t-\tau)}{\left(\left(-z+\frac{t-\tau}{\alpha}\right)^{2}+\rho^{2}\right)^{3 / 2}}\right\}\right]
$$

$e_{\varphi}(t)=-b \mu \frac{I_{0}}{\tau}\left[\frac{\rho}{\left(z^{2}+\rho^{2}\right)^{3 / 2}}(u(t)-u(t-\tau))-\frac{\rho u(t)}{\left((-z+t / \alpha)^{2}+\rho^{2}\right)^{3 / 2}}+\frac{\rho u(t-\tau)}{\left(\left(-z+\frac{t-\tau}{\alpha}\right)^{2}+\rho^{2}\right)^{3 / 2}}\right]$.

It should be noted that the calculation of eq (11) is facilitated by the use of the shifting theorem [4]. The components of the magnetic field may be calculated from eqs (1) and (11). They are:

$$
\begin{aligned}
h_{\rho}^{(t)}=b \frac{I_{0}}{\tau}\left[\frac{3 \rho z(t u(t)-(t-\tau) u(t-\tau))}{\left(z^{2}+\rho^{2}\right)^{5 / 2}}+\frac{\alpha \rho}{2}\left\{\frac{u(t)-u(t-\tau)}{\left(z^{2}+\rho^{2}\right)^{3 / 2}}\right.\right. & \left.\left.-\frac{u(t)}{\left((-z+t / \alpha)^{2}+\rho^{2}\right)^{3 / 2}}+\frac{u(t-\tau)}{\left(\left(-z+\frac{t-\tau}{\alpha}\right)^{2}+\rho^{2}\right)^{3 / 2}}\right\}\right] \\
h_{z}^{(t)}=b \frac{I_{0}}{\tau}\left[\frac{3 z^{2}(t u(t)-(t-\tau) u(t-\tau))}{\left(z^{2}+\rho^{2}\right)^{5 / 2}}+\frac{\alpha}{2}\left\{\frac{-z u(t)-u(t-\tau)}{\left(z^{2}+\rho^{2}\right)^{3 / 2}}\right.\right. & \\
& \left.\left.-\frac{(-z+t / \alpha) u(t)}{\left((-z+t / \alpha)^{2}+\rho^{2}\right)^{3 / 2}}+\frac{u(t-\tau)\left(-z+\frac{t-\tau}{\alpha}\right)}{\left(\left(-z+\frac{t-\tau}{\alpha}\right)^{2}+\rho^{2}\right)^{3 / 2}}\right\}\right] .
\end{aligned}
$$


The components of the electric and magnetic fields, eqs (12), (13), and (14) may be decomposed into more elementary units whose physical significance will be explained. In particular,

$$
\begin{aligned}
& h_{\rho}(t)=A_{\rho}(t)+B_{\rho}(t)+C_{\rho}(t)+D_{\rho}(t) \\
& h_{z}(t)=A_{z}(t)+B_{z}(t)+C_{z}(t)+D_{z}(t) \\
& e_{\phi}(t)=B_{\phi}(t)+C_{\phi}(t)+D_{\phi}(t)
\end{aligned}
$$

where

$$
\begin{aligned}
& A_{\rho}(t)=b \frac{I_{0}}{\tau} \frac{3 \rho z}{\left(z^{2}+\rho^{2}\right)^{5 / 2}}[t u(t)-(t-\tau) u(t-\tau)] \\
& B_{\rho}(t)=b \frac{I_{0}}{\tau} \frac{\alpha \rho}{2} \frac{u(t)-u(t-\tau)}{\left(z^{2}+\rho^{2}\right)^{3 / 2}} \\
& C_{\rho}(t)=-b \frac{I_{0}}{\tau} \frac{\alpha \rho}{2} \frac{u(t)}{\left((-z+t / \alpha)^{2}+\rho^{2}\right)^{3 / 2}} \\
& D_{\rho}(t)=b \frac{I_{0}}{\tau} \frac{\alpha \rho}{2} \frac{u(t-\tau)}{\left(\left(-z+\frac{t-\tau}{\alpha}\right)^{2}+\rho^{2}\right)^{3 / 2}} \\
& A_{z}(t)=b \frac{I_{0}}{\tau} \frac{3 z^{2}}{\left(z^{2}+\rho^{2}\right)^{5 / 2}}[t u(t)-(t-\tau) u(t-\tau)] \\
& B_{z}(t)=b \frac{I_{0}}{\tau} \frac{\alpha}{2} \frac{(-z)[u(t)-u(t-\tau)]}{\left(z^{2}+\rho^{2}\right)^{3 / 2}} \\
& C_{z}(t)=-b \frac{I_{0}}{\tau} \frac{(-z+t / a) u(t)}{\left((-z+t / \alpha)^{2}+\rho^{2}\right)^{3 / 2}} \\
& D_{z}(t)=b \frac{I_{0}}{\tau} \frac{u(t-\tau)\left(-z+\frac{t-\tau}{\alpha}\right)}{\left(\left(-z+\frac{t-\tau}{\alpha}\right)^{2}+\rho^{2}\right)^{3 / 2}} \\
& B_{\phi}(t)=-\mu b \frac{I_{0}}{\tau} \frac{\rho}{\left(z+\rho^{2}\right)^{3 / 2}}[u(t)-u(t-\tau)] \\
& C_{\phi}(t)=\mu b \frac{I_{0}}{\tau} \frac{\rho u(t)}{\left((-z+t / \alpha)^{2}+\rho^{2}\right)^{3 / 2}} \\
& D_{\phi}(t)=-\mu b \frac{I_{0}}{\tau} \frac{\rho u(t-\tau)}{\left(\left(-z+\frac{t-\tau}{\alpha}\right)^{2}+\rho^{2}\right)^{3 / 2}} .
\end{aligned}
$$

The significance of these terms may be explained as follows:

The $A(t)$ terms may be regarded as primary field terms. They represent the fields that would be produced in the absence of the conducting sheet. Their time behavior is the same as that of the source.

The $B(t), C(t)$, and $D(t)$ terms represent surges resulting from the presence of the sheet. A closer examination of $B(t), C(t)$, and $D(t)$ reveals the following:

$B(t)$ and $D(t)$ have the same signs but act in different time intervals. $B(t)$ acts in the interval $0 \leq t \leq \tau$, i.e., during the rise of the excitation, while $D(t)$ acts in the interval $\tau \leq t<\infty$, i.e., after the excitation has reached its peak value. 
The $C(t)$ terms are opposite in sign to the $B(t)$ and $D(t)$ terms and are smaller in magnitude for $t>0$. They may be regarded as cancellation terms which modify the surge terms $B(t)$ and $D(t)$. An examination of eqs (12), (13), and (14) shows that the $C(t)$ terms decrease monotonically in the interval $0 \leq t \leq \tau$. Since the $B(t)$ terms are constant in the interval $0 \leq t \leq \tau$, the cancellation becomes progressively weaker during the rise of the excitation. The surges reach their maxima at time $t=\tau$, since the cancellation is weakest here. At this time the total fields are

$$
\begin{gathered}
h_{\rho}(\tau)=b I_{0}\left[\frac{3 \rho z}{\left(\rho^{2}+z^{2}\right)^{5 / 2}}+\frac{\alpha \rho}{2}\left\{\frac{1}{\left(z^{2}+\rho^{2}\right)^{3 / 2}}-\frac{1}{\left((-z+\tau / \alpha)^{2}+\rho^{2}\right)^{3 / 2}}\right\}\right] \\
h_{z}(\tau)=b I_{0}\left[\frac{3 z^{2}}{\left(\rho^{2}+z^{2}\right)^{5 / 2}}+\frac{\alpha}{2}\left\{\frac{-z}{\left(z^{2}+\rho^{2}\right)^{3 / 2}}-\frac{(-z+\tau / \alpha)}{\left((-z+\tau / \alpha)^{2}+\rho^{2}\right)^{3 / 2}}\right\}\right] \\
e_{\phi}(\tau)=-\mu b I_{0}\left[\frac{\rho}{\left(z^{2}+\rho^{2}\right)^{3 / 2}}-\frac{\rho}{\left((-z+\tau / \alpha)^{2}+\rho^{2}\right)^{3 / 2}}\right]
\end{gathered}
$$

After $t=\tau, B(t)$ is replaced by $D(t) . \quad C(t) \rightarrow 0$ and $D(t) \rightarrow 0$ in such a way that the surge fields die out and only the primary fields remain.

An examination of eqs (15), (16), and (17) shows that the surge in $h_{p}$ is opposite in sign to the primary field (for $z<0$ ) and thus serves to reduce it. The surge in $h_{z}$, on the other hand, is the same as that of the primary field and thus increases the total field. The electric field term $e \phi$ consists only of a surge. The primary field is zero. It can be seen, in terms of the parameter $\tau / \alpha$, that the cancellation term $C(t)$ will be reduced for large values of $\tau / \alpha$ and thus the surge will have a greater effect for longer rise times and for thinner or more poorly conducting sheets. The low-frequency approximation on which these results are based restricts these considerations to values $\tau|\alpha\rangle\rangle z$ and does not permit a discussion of small rise times or thick conducting sheets.

Attempts to deal with thicker sheets have led to complicated integrals. Other authors such as Wait [3], Lowndes [5], Bhattacharyya [6], Gordon [7], and Price [8] have attempted to integrate expressions involving rational functions and exponential arising in such problems. They have been forced to make highly restrictive assumptions. Further work in this direction would appear to be desirable.

The transient response of a horizontal dipole is similar to that of the loop.

The author thanks Professor R. W. P. King for calling this problem to his attention and for many helpful discussions.

\section{References}

[1] J. R. Wait, Shielding of a transient electromagnetic dipole field by a conducting sheet, Can. J. Phys. 34, 890 (1956).

[2] J. R. Wait, Induction in a conducting sheet by a small current-carrying loop, Appl. Sci. Research B3, 230 (1953).

[3] J. R. Wait, The magnetic dipole over the horizontally stratified earth, Can. J. Phys. 29, 577 (1951).

[4] B. van der Pol and H. Bremmer, Operational calculus (Cambridge University Press, Cambridge, England, $1950)$.

[5] J. S. Lowndes, A transient magnetic dipole source above a two-layer earth, Quart. J. Mech. Appl. Math. 10, 79 (1957).

[6] B. K. Bhattacharyya, Electromagnetic induction in a two-layer earth, J. Geophys. Research 60, 279 (1955).

[7] A. N. Gordon, The field induced by an oscillating magnetic dipole outside a semi-infinite conductor, Quart. J. Mech. Appl. Math. 4, 106 (1951).

[8] A. T. Price, Electromagnetic induction in a semi-infinite conductor with a plane boundary, Quart. J. Mech. Appl. Math. 3, 385 (1950).

(Paper 64D5-94) 